\title{
Response Factors Enable Rapid Quantitative 2D NMR Analysis in Catalytic Biomass Conversion to Renewable Chemicals
}

\author{
Elliot, Samuel Gilbert; Tosi, Irene; Riisager, Anders; Taarning, Esben; Meier, Sebastian
}

Published in:

Topics in Catalysis

Link to article, DOI:

10.1007/s11244-019-01131-y

Publication date:

2019

Document Version

Peer reviewed version

Link back to DTU Orbit

Citation (APA):

Elliot, S. G., Tosi, I., Riisager, A., Taarning, E., \& Meier, S. (2019). Response Factors Enable Rapid Quantitative 2D NMR Analysis in Catalytic Biomass Conversion to Renewable Chemicals. Topics in Catalysis, 62(7-11), 580598. https://doi.org/10.1007/s11244-019-01131-y

\section{General rights}

Copyright and moral rights for the publications made accessible in the public portal are retained by the authors and/or other copyright owners and it is a condition of accessing publications that users recognise and abide by the legal requirements associated with these rights.

- Users may download and print one copy of any publication from the public portal for the purpose of private study or research.

- You may not further distribute the material or use it for any profit-making activity or commercial gain

- You may freely distribute the URL identifying the publication in the public portal 


\title{
Response Factors Enable Rapid Quantitative 2D NMR Analysis in Catalytic Biomass Conversion to Renewable Chemicals
}

\author{
Samuel G. Elliot, ${ }^{\mathrm{la} *}$ Irene Tosi, ${ }^{\mathrm{b}}$ Anders Riisager, ${ }^{\mathrm{lc}}$ Esben Taarning, ${ }^{2}$ and Sebastian Meier ${ }^{\mathrm{a} *}$ \\ 'Technical University of Denmark, Department of Chemistry, Kemitorvet, 2800-Kgs. Lyngby, Denmark \\ 2Haldor Topsøe A/S, Haldor Topsøes Alle 1, 2800-Kgs. Lyngby, Denmark \\ **sgiel@kemi.dtu.dk, ORCID ID: 0000-0002-3022-8060. \\ 'ORCID ID: 0000-0002-2486-6971. \\ ORCID ID: 0000-0002-7086-1143. \\ d* semei@kemi.dtu.dk, ORCID ID: 0000-00033761-3217.
}

\begin{abstract}
Carbohydrate conversion offers access to a variety of chemicals with diverse functionalities. An accurate analysis of the multiple products in post-reaction material is indispensable for enabling good atom economy in biorefining. A certain need for reconsidering current analytical approaches to chemocatalytic biomass conversion is witnessed by the often poor carbon balances that are reported for carbohydrate conversion processes. Carbohydrate conversion usually includes isomerization and/or dehydration, therefore analytical approaches that are suitable for the distinction and concurrent quantification of isomers are desirable for developing sustainable processes towards known and new chemicals. Quantitative 1D NMR spectroscopy can be used to determine absolute concentrations in the absence of purified reference compounds and can thereafter be used to obtain response factors in other analytical methods resolving the compounds of interest. Here, we show that this approach is applicable for obtaining response factors relative to an internal standard for rapid, highly resolved 2D NMR spectra at natural isotopic abundance. Following calibration, this approach provides a limit of quantification in the order of $0.8 \mathrm{mM}$ within an experiment time of a few minutes. The approach is particularly beneficial for the quantification of compounds at low concentrations, for instance in initial rate experiments, and for the quantification of low populated reaction intermediates.
\end{abstract}

\section{Keywords}

biomass, catalysis, qNMR, quantitative analysis, reference standard, response factor

Electronic supplementary material The online version of this article (https://doi.org/XXX) contains supplementary material, which is available to authorized users. 


\section{Introduction}

The current trajectories of global population growth and of natural resource consumption are unsustainable, as many natural resources are poised to deplete within the next few generations. Hence, sustainable means of producing known and new chemicals to sustain our materials- and energydemanding lifestyles are needed. In this shift to alternative industries that rely more on renewables and less on petroleum refining, biomass is a promising feedstock $[1,2]$. Biomass conversion offers access to a variety of chemicals with diverse functionalities, for instance through the use of solid acid catalysts such as zeolites $[3,4]$.

Accurate qualitative and quantitative analysis of the multiple products in post-reaction material is indispensable for the development of biorefinery technology. The conversion of biomass often includes the isomerization or dehydration of carbohydrates [3,5-10]. Therefore, analytical approaches that are suitable for the distinction and concurrent quantification of isomers (such as monosaccharides and their respective dehydration products) are poised to be particularly valuable for the analysis of biomass conversion products, and thus for aiding sustainable processes towards known and new chemicals. A certain need for reconsidering analytical approaches to chemocatalytic biomass conversion is witnessed by the often poor yields and low carbon balances that are reported for carbohydrate conversion processes, with little effort to identify and quantify the byproduct "dark matter" in the mixture [11].

NMR spectroscopy has been used in qualitative and quantitative analysis for more than five decades [12]. Recent improvements in instrumentation enable the determination of compound structures also in complex product mixtures $[10,13,14]$. At the same time, the quantitative use of NMR (qNMR) has gained importance in metabolic studies [15-18], natural products research $[19,20]$, food analysis $[21,22]$ and pharmaceutical research [18,23-25]. Compound identification and quantification in mixtures has gained much attention in biocatalysis, while applications in chemocatalysis have so far remained sparse [26-29]. Wider implementation of NMR methodology in chemocatalysis could be attractive, considering that both the substrate (mostly carbohydrates) and the interest in catalyst function and dysfunction bear close resemblance in metabolic and chemocatalytic processes.

Recently, we have shown that quantitative ${ }^{13} \mathrm{C}$ NMR permits the identification of novel chemicals, and optimization of their formation by solvent variation in the stannosilicate catalyzed conversion of carbohydrates [14]. Owing to its superior resolution over ${ }^{1} \mathrm{H}$ NMR, ${ }^{13} \mathrm{C}$ NMR has an important role to play in the analysis of complex mixtures containing chemically similar compounds. This approach permits improved determination of carbon balances and of factors affecting product distribution upon biomass conversion.

Quantitative ${ }^{13} \mathrm{C}$ NMR spectroscopy suffers from mediocre sensitivity due to the low natural abundance and the lower magnetogyric ratio of ${ }^{13} \mathrm{C}$ as compared especially to ${ }^{1} \mathrm{H}$. The mediocre sensitivity of ${ }^{13} \mathrm{C}$ NMR results in lengthy experiment times that challenge high throughput applications of the methodology. In contrast, faster and more sensitive quantitative ${ }^{1} \mathrm{H}$ NMR methods result in much more congested NMR spectra as the ${ }^{1} \mathrm{H}$ NMR chemical shift range is approximately 20 -fold smaller than the ${ }^{13} \mathrm{C}$ chemical shift range. In addition, ${ }^{1} \mathrm{H}$ signals are split into multiplets due to scalar coupling to other protons in the same molecule, thus further congesting the ${ }^{1} \mathrm{H}$ NMR spectrum. Here, we show that workarounds to these problems can be achieved by indirect detection of ${ }^{13} \mathrm{C}$ signals through highly resolved two-dimensional ${ }^{1} \mathrm{H}^{-13} \mathrm{C}$ HSQC spectra. These ${ }^{1} \mathrm{H}-{ }^{13} \mathrm{C}$ HSQC spectra are then used for compound quantification upon an initial determination of response factors in the ${ }^{1} \mathrm{H}-{ }^{13} \mathrm{C}$ HSQC relative to quantitative ${ }^{13} \mathrm{C}$ NMR spectra. To this end, normalization to a stable internal quantification standard was performed (Scheme 1). Two-dimensional ${ }^{1} \mathrm{H}-{ }^{13} \mathrm{C}$ HSQC spectra were subsequently optimized for high throughput, achieving a limit of quantification (LOQ) in the order of $0.8 \mathrm{mM}$ with only 5 minutes of experiment time per sample. When employing a $0.4 \mathrm{M}$ carbohydrate feedstock, this accuracy translates to a LOQ of $\leq 0.2$ mol\% carbon. Such high accuracy can be beneficial in various settings, such as initial rate experiments under low conversion or in the tracking of pathway intermediates [26,27]. 


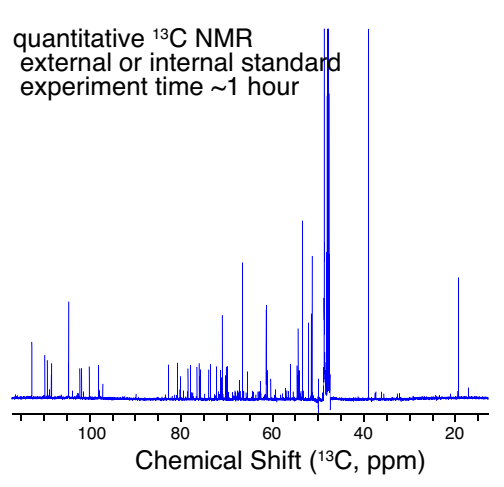

Analyte signal relative to standard

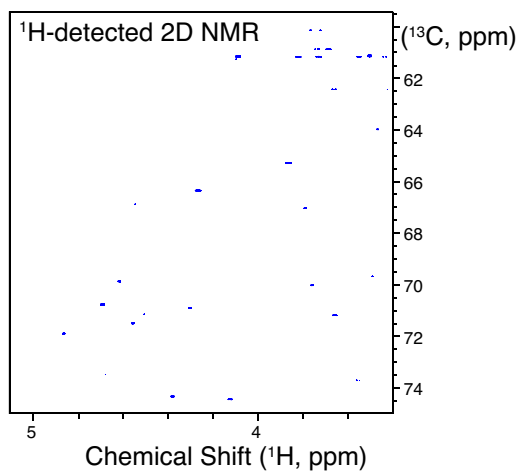

Analyte signal relative to standard

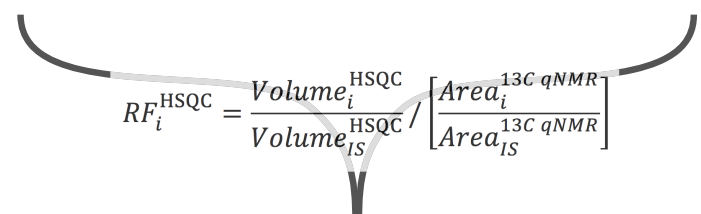

Response factor for quantitative 2D NMR experiment time $<10$ minutes lower limit of quantification $0.8 \mathrm{mM}$

Scheme 1. Strategy for the rapid and accurate quantitative analysis of reaction mixtures using response factors in $2 \mathrm{D}$ NMR.

\section{Results and Discussion}

\subsection{Internal Standards}

Internal or external standards are employed in qNMR to obtain highly accurate and precise results $[25,30]$. Internal standardization removes many experimental uncertainties and is considered superior to external standardization [30,31]. The internal standards can be added either prior to biomass conversion or subsequent to the reaction, each approach having advantages and disadvantages. Many biomass reactions are conducted above the boiling point of the solvent, which is typically low in order to expedite subsequent purification steps. These temperatures imply possible solvent boil-off with concomitant changes to analyte concentrations, thus arguing for addition of internal standard into the reaction medium prior to the reaction. This approach was therefore employed here.

Special precautions have to be taken in to account for the use of internal standards during the reaction: the standards should not decompose at high temperatures (often between 100 and $200{ }^{\circ} \mathrm{C}$ ) and should not contain acidic or other functional groups that may affect the reaction system. Prospective qNMR standard substances with Brønsted acidic or basic sites were excluded from the study (for instance the commonly employed standard maleic acid). Beyond being chemically inert, suitable standards yield strong ${ }^{13} \mathrm{C}$ signals at low concentration that do not overlap with alcoholic and olefinic carbon positions that are common in carbohydrate derived products. To this avail, a minimal number of NMR signals are desirable, simplifying analysis and reducing risk of spectral overlap. Strong signals can be warranted by the presence of protonated carbons with relaxation times comparable to carbons in the analyte mixture and by symmetric molecules, where several magnetically equivalent carbons contribute the signal. For instance, dimethyl sulfoxide and dimethyl sulfone contain two equivalent carbons, thus doubling signal intensity, while mesitylene contains three sets of three equivalent carbons and 1,4-dioxane contains four equivalent carbons also increasing the intensity (Scheme 2).<smiles>Cc1cc(C)cc(C)c1</smiles>

Mesitylene<smiles>CS(C)=O</smiles><smiles>C1COCCO1</smiles><smiles>OCC(O)C(O)C(O)CO</smiles>

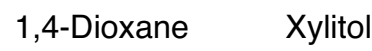<smiles>CS(C)(=O)=O</smiles><smiles>OCC(O)CO</smiles>

Dimethylsulfoxide Dimethylsulfone Glycerol

Scheme 2. Structures of selected standard compounds tested herein.

In addition, a high boiling point was deemed preferable to ease preparation and reduce risk of material loss during the reaction. Finally, reference compounds should be available in high purity at low cost, be non-hazardous and be miscible with 
industrially relevant (and especially green) solvents $[24,25]$. Based on these considerations, the standards of Scheme 2 and Table 1 were selected.

Table 1. Overview of selected internal standards and their performance on conditions 1 and 2.

\begin{tabular}{lllcc}
\hline \multirow{2}{*}{ Entry } & \multirow{2}{*}{ Internal Standard } & B.P. & Number of NMR signals \\
\cline { 5 - 5 } & & {$\left[{ }^{\circ} \mathbf{C}\right]$} & ${ }^{\mathbf{1}} \mathbf{H}^{\boldsymbol{a}}$ & ${ }^{\mathbf{1 3}} \mathbf{C}$ \\
\hline 1 & Mesitylene & 164 & 2 & 3 \\
\hline 2 & 1,4-dioxane & 101 & 1 & 1 \\
\hline 3 & Dimethyl sulfoxide & 189 & 1 & 1 \\
\hline 4 & Dimethyl sulfone & $107^{b}$ & 1 & 1 \\
\hline 5 & Glycerol & 290 & 3 & 2 \\
\hline 6 & Xylitol & $94^{b}$ & 3 & 3 \\
\hline${ }^{a}$ Considering only protons attached to carbon \\
${ }^{b}$ Melting point
\end{tabular}

The miscibility or solubility of the prospective standard compounds was assessed with water, methanol, chloroform and dimethyl sulfoxide as solvents (Supplementary Table S1). Xylitol showed limited solubility in all solvents except water and was not pursued further. In contrast, mesitylene was very poorly miscible with water, but miscible with the other four solvents. Mesitylene, 1,4-dioxane, dimethyl sulfoxide, dimethyl sulfone and glycerol were thus all deemed potentially suitable primary standards for quantitative NMR.

\subsection{Reaction Performance}

A test reaction was selected to evaluate the stability of internal standards in the presence of Lewis acidic zeolite Sn-Beta. The test reaction was based on previous work within biomass conversion and was comprised of $8 \mathrm{wt} \%$ xylose and $2 \mathrm{wt} \%$ Sn-Beta zeolite catalyst in methanol [32]. The mixture was reacted at $160^{\circ} \mathrm{C}$ for up to 24 hours in order to evaluate the stability of internal quantification standards on this timescale. Full conversion was already achieved after 1-2 hours. Figure 1 shows the recovery of the standards with reasonable solubility, i.e. mesitylene, 1,4-dioxane, dimethyl sulfoxide (DMSO), dimethyl sulfone $\left(\mathrm{DMSO}_{2}\right)$ and glycerol. Recovery indicated that all compounds were stable over 2 hours under the reaction conditions, while DMSO showed degradation on longer timescales. Dioxane, $\mathrm{DMSO}_{2}$ mesitylene and glycerol thus appeared particularly suitable as internal standards in the presence of Lewis acidic catalysts for prolonged reaction times. For short reaction times, as those required for full conversion in our test reaction, all of the selected reference standards appeared suitable and sufficiently stable as internal standards.

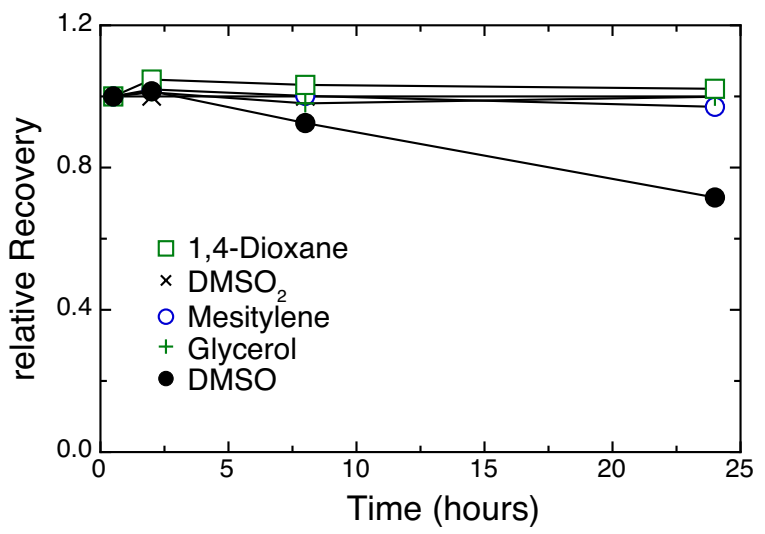

Figure 1. Stability of internal standards over time. The recovery of NMR signal for five compounds is displayed after $0.5,2,8$, and 24 hours.

\subsection{Determination of 2D NMR Responses Relative to 1D qNMR}

Biomass conversion can lead to new chemicals that do not exist as commercial compounds for the determination of response factors in analyses. Quantitative 1D NMR analyses can be used to determine the amounts of chemicals without the need for purified and isolated compounds, as concentrations can be determined relative a chemically non-identical standard [33]. The signal response in quantitative 1D NMR (e.g. ${ }^{13} \mathrm{C} \mathrm{NMR}$ ) is proportional to the concentration of atoms generating the signal and is therefore identical even for different compounds. Quantitative 1D NMR spectra have thus previously been used in order to normalize chromatographic analyses in instances where purified and isolated reference compounds are not available [33]. NMR spectroscopy, however, offers an even more versatile toolbox of multi-dimensional spectra with advantageous spectral resolution and sensitivity. ${ }^{1} \mathrm{H}-{ }^{13} \mathrm{C}$ HSQC spectra, as are employed here, provide 10 -fold higher sensitivity than ${ }^{13} \mathrm{C}$ NMR spectra and additional resolution due to the acquisition of two spectral dimensions (Supplementary Table S2).

Here, we acquired quantitative ${ }^{13} \mathrm{C}$ NMR spectra and ${ }^{1} \mathrm{H}-{ }^{13} \mathrm{C}$ HSQC spectra on a reaction mixture, derived from the aforementioned Sn-Beta catalysed conversion of xylose in methanol at $160^{\circ} \mathrm{C}$. Four products were selected as targets for proof of principle, Scheme 3, only one of which was readily available commercially. The targets all possess secondary alcohols, producing signals within a similar spectral region. Signals in the secondary alcohol region in a ${ }^{1} \mathrm{H}^{-13} \mathrm{C}$ HSQC (of 24 minutes duration) are displayed in Figure 2. 
<smiles>COC(=O)C(O)/C=C/CO</smiles>

(trans-2, 5-dihydroxy-3pentenoic acid methyl ester)<smiles>C=CC(O)C(=O)OC</smiles>

MVG (methyl vinyl glycolate)<smiles>CCCC(O)C(=O)OCC</smiles>

(methyl 2-hydroxy-4methoxybutanoate)<smiles>COC(=O)C(C)O</smiles>

$\mathrm{ML}$ (methyl lactate)

Scheme 3. Selection of major products from Lewis acid catalyzed conversion of xylose, employed for the procedures in this work.

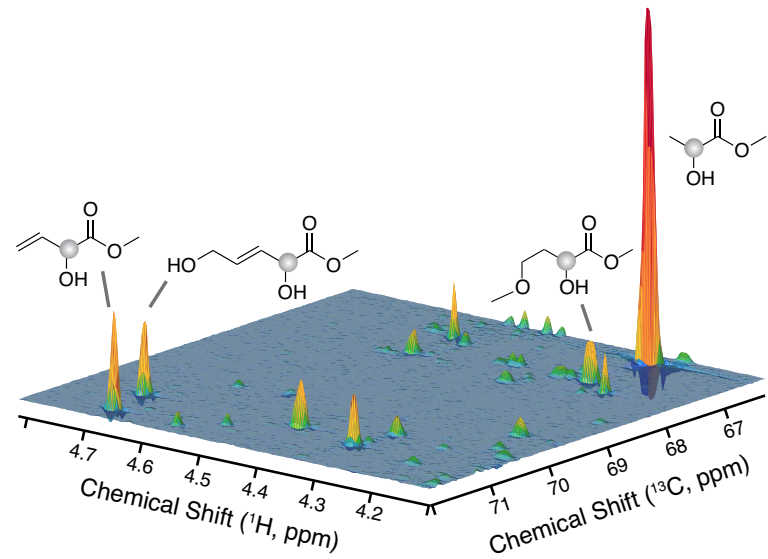

Figure 2. The hydroxyl region of the ${ }^{1} \mathrm{H}-{ }^{13} \mathrm{C}$ HSQC spectra with a selection of identified products indicated.

Following identification of the signals as in Figure 2, the signal areas from quantitative ${ }^{13} \mathrm{C}$ NMR spectra can be employed to quantify the target compounds. Provided that the amount of internal standard (IS) is known, a compound $i$ can be quantified using equation 1 , where $N$ is the number of carbons in the molecule producing the signal, and $c_{i}$ and $c_{I S}$ are the concentrations of analyte $i$ and internal standard, respectively.

$$
\text { (1) } \frac{\operatorname{Area}_{\mathrm{i}}^{13 C \text { qNMR }} / N_{i}^{\text {Carbon }}}{\operatorname{Area}_{\mathrm{IS}}^{13 C N M R} / N_{I S}^{\text {Carbon }}}=\frac{c_{i}}{c_{I S}} \text {, }
$$

Signal areas relative to the internal standard (DMSO) in quantitative ${ }^{13} \mathrm{C}$ NMR spectra (i.e. analyte concentrations) were correlated to the corresponding signal volumes in ${ }^{1} \mathrm{H}-{ }^{13} \mathrm{C}$ HSQC spectra, as shown in Figure 3, yielding compound specific response factors based on the $\mathrm{C} 2-\mathrm{H} 2$ group. The response factor $R F_{i}^{2 D N M R}$ of a selected signal in compound $j$ is here defined as:

(2) $R F_{i}^{\mathrm{HSQC}}=\frac{\text { Volume }_{i}^{\mathrm{HSQC}}}{\text { Volume }_{I S}^{\mathrm{HSQC}}} /\left[\frac{\text { Area }_{i}^{13 C \text { qNMR }}}{\text { Area }_{I S}^{13 C q N M R}}\right]$

In plots of HSQC signal volumes $v s$ quantitative ${ }^{13} \mathrm{C}$ NMR signal areas each relative to the standard, the response factors thus simply correspond to the slope. In order to assess the accuracy of response factor determinations, the above-mentioned reaction was conducted for different durations to achieve a variety of concentrations for the target compounds. Linear regressions yielded Pearson correlation coefficients above 0.997 for each of the four displayed determinations (Figure 3), demonstrating excellent consistency of $1 \mathrm{D}$ and 2D NMR quantifications.

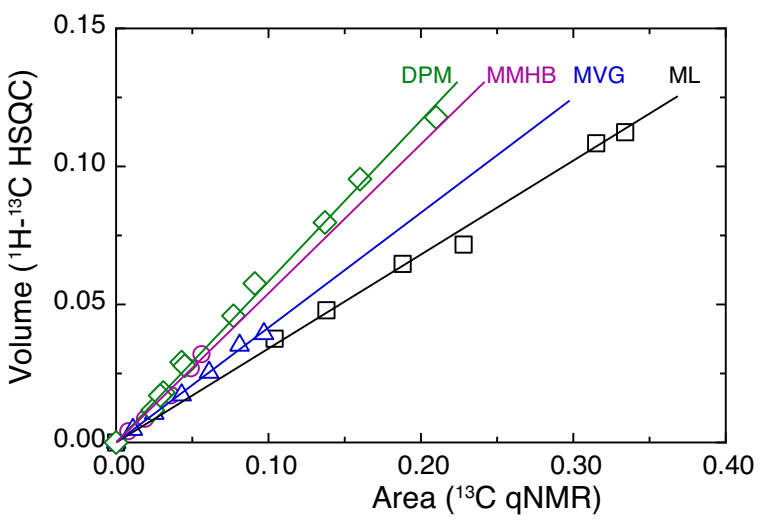

Figure 3. Correlations between integrals from ${ }^{1} \mathrm{H}-{ }^{13} \mathrm{C}$ HSQC normalised to the internal standard (DMSO) integral and quantitative ${ }^{13} \mathrm{C}$ NMR integrals normalised to internal standard. Correlations and linear regressions are shown for four selected products of the Sn-Beta catalysed xylose conversion in methanol.

By substitution of equation 2 into equation 1 , an equation for determining the concentration by ${ }^{1} \mathrm{H}-{ }^{13} \mathrm{C}$ HSQC signal areas instead of quantitative 1D ${ }^{13} \mathrm{C}$ NMR is obtained:

(3) $c_{i}=\frac{\text { Volume }_{\mathrm{i}}^{\mathrm{HSQC}}}{\text { Volume }_{\mathrm{IS}}^{\mathrm{HSQC}}} \times c_{I S} \times \frac{N_{I S}^{\text {Carbon }}}{N_{i}^{\text {Carbon }}} / R F_{i}^{\mathrm{HSQC}}$

As ${ }^{1} \mathrm{H}-{ }^{13} \mathrm{C}$ HSQC spectra are more sensitive than quantitative ${ }^{13} \mathrm{C}$ NMR spectra, equation 3 permits more accurate quantification of the compound $i$ and faster determinations than was possible by ${ }^{13} \mathrm{C}$ NMR alone (Table S2 indicates $10-$ fold lower limit of detection at 11-fold faster analyses). Conversion of concentrations to carbon yields requires qualitative knowledge of the chemical identity of compounds $i$. Identification of the compounds can be performed in unpurified reaction mixtures using spectra of reference compounds 
$[10,34]$ or through multidimensional assignment spectra recorded with high-field NMR $[10,13,14]$.

\subsection{Variation of Response Factors}

Various factors affect the signal response in multidimensional NMR spectra. Magnetization transfer can vary due to variable scalar coupling constants and loss of transverse magnetization during the transfer can likewise vary between molecules. Both of these factors are expected to play very minor roles for the response factors determined in the experiments of Figure 3. Scalar ${ }^{1} J_{\mathrm{C} 2 \mathrm{H} 2}$ couplings were determined for $\alpha$-hydroxy esters and showed a very narrow distribution of couplings constants in the analogous structural motifs (variation between 146.0 and $146.7 \mathrm{~Hz}$, Figure 4A). Loss of transverse magnetization during the transfer step of few milliseconds in ${ }^{1} \mathrm{H}-{ }^{13} \mathrm{C}$ HSQC is likewise expected to be negligible.

The ${ }^{1} \mathrm{H}_{-}{ }^{13} \mathrm{C}$ HSQC spectra were recorded with a short inter-scan recycle delay in order to minimize the experiment time of the spectra. Accordingly, response factors show size dependence with higher response for the slightly larger compounds, as longitudinal $\left(T_{1}\right)$ relaxation in these compounds is more complete during the inter-scan delay. The predominant effect of inter-scan $T_{1}$ relaxation on differential response factors was probed by acquiring long HSQC spectra with 15 seconds instead of 1 second inter-scan relaxation delays. Response factors in these experiments were more homogeneous than for experiments using a 1 second recycle delay (Figure 5). Response factors generally approached a value near 0.33 for longer recycle delays, consistent with the transfer of polarization to carbon from three protons in the DMSO methyl group, but from only one proton in the $\alpha$-hydroxy ester $\mathrm{C} 2-\mathrm{H} 2$ group. These findings indicate that response factors may be predicted from molecular weight and molecular weight of unknown compounds estimated from the response factor. Alternatively, the response factor for different compounds can be equalised, through suitable adjustments to the 2D NMR experiment, if desired.
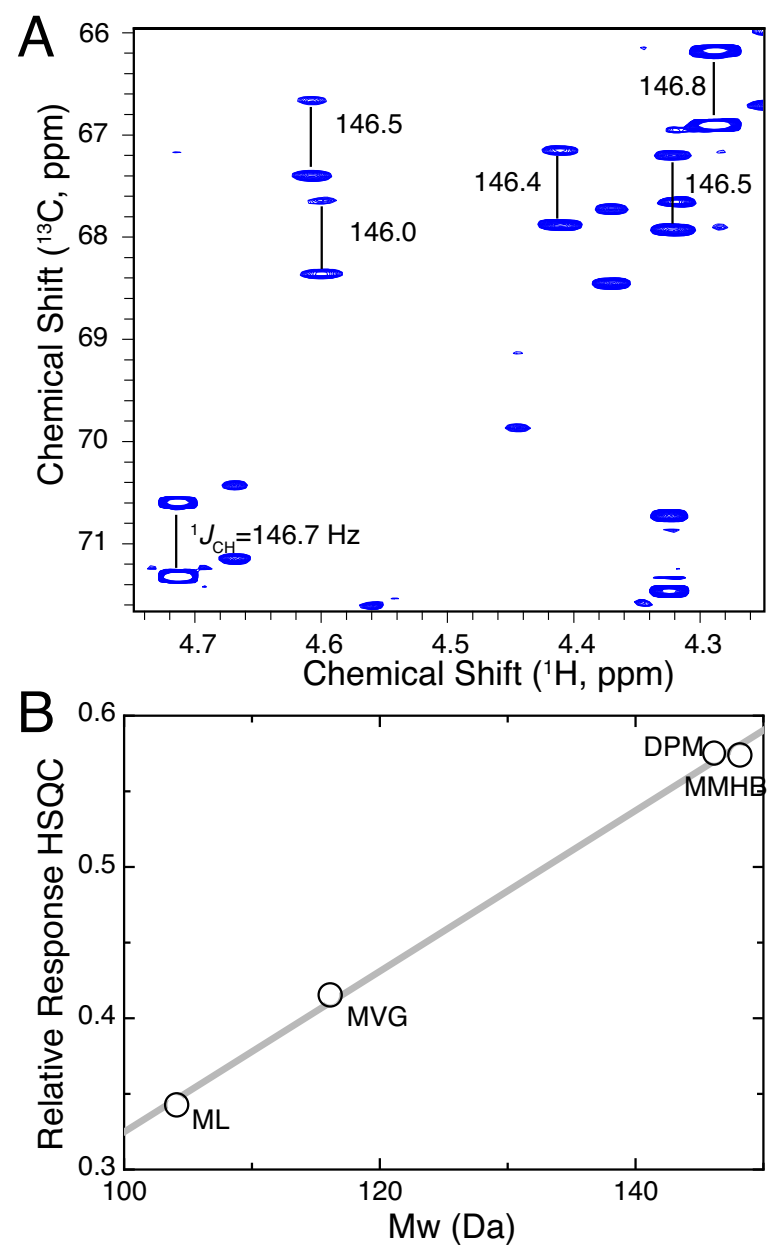

Figure 4. (A) Contour plot of a ${ }^{1} \mathrm{H}-{ }^{13} \mathrm{C}-\mathrm{HSQC}$ spectrum recorded without ${ }^{1} \mathrm{H}$ decoupling during the ${ }^{13} \mathrm{C}$ evolution time. Only small variation (and highly similar ${ }^{1} \mathrm{H}^{-13} \mathrm{C}$ magnetization transfer efficiency) exists for different $\alpha$-hydroxy esters. (B) Response factor as a function of molecular weight when using inter-scan recycle delays of 1 second.

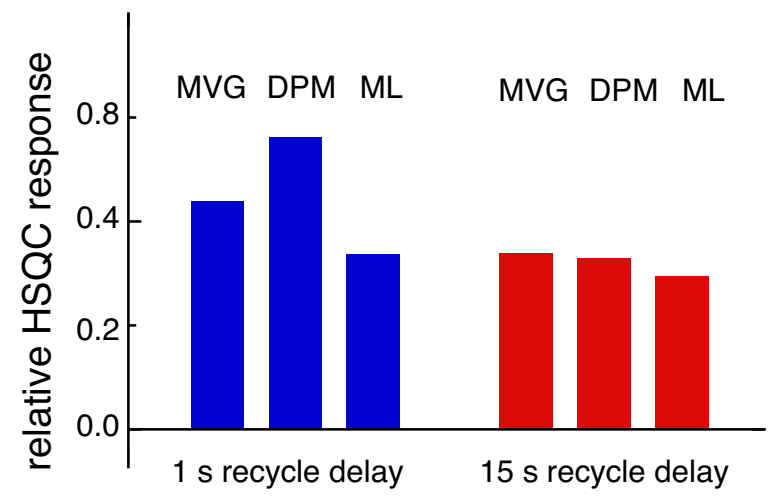

Figure 5. ${ }^{1} \mathrm{H}-{ }^{13} \mathrm{C}$ HSQC response factors for the indicated $\alpha$ hydroxyesters when using inter-scan recycle delays of 1 second and 15 seconds, respectively. Complete ${ }^{1} \mathrm{H}$ relaxation leads to more homogeneous response factors near 0.33 relative to DMSO. 


\subsection{Accelerated Data Acquisition}

The results above showed that upon calibration with few samples, responses in ${ }^{1} \mathrm{H}-{ }^{13} \mathrm{C}$ HSQC spectra can be used to quantitatively determine mixture composition in biomass conversion with sensitivity and throughput that is superior to the use of quantitative ${ }^{13} \mathrm{C}$ NMR. The use of response factors permits the rapid acquisition of two-dimensional spectra with short inter-scan relaxation delays. Twodimensional spectra can be further accelerated by recent methodological advances permitting data acquisition through sampling of only a fraction of the data points that were conventionally acquired. Spectra acquired in the conventional manner and through the non-uniform sampling of the ${ }^{13} \mathrm{C}$ dimension are displayed in Figure 6. The spectrum in Figure $6 \mathrm{~B}$ recorded only $20 \%$ of the data points that were acquired in Figure 6A and hence could be acquired in only 5 minutes.

Spectral quality in the spectrum using nonuniform sampling was still acceptable without any disturbance to signal volumes or introduction of overlapping artefacts. The LOQ was only marginally affected, increasing from $0.75 \mathrm{mM}$ in the regular 24 minute experiment to $0.83 \mathrm{mM}$ using non-uniform sampling (Supplementary Table S2). Thus, accurate quantitative determinations can be obtained with minimal sample preparation and within few minutes, a timescale competitive with most other commonly employed analysis methods.

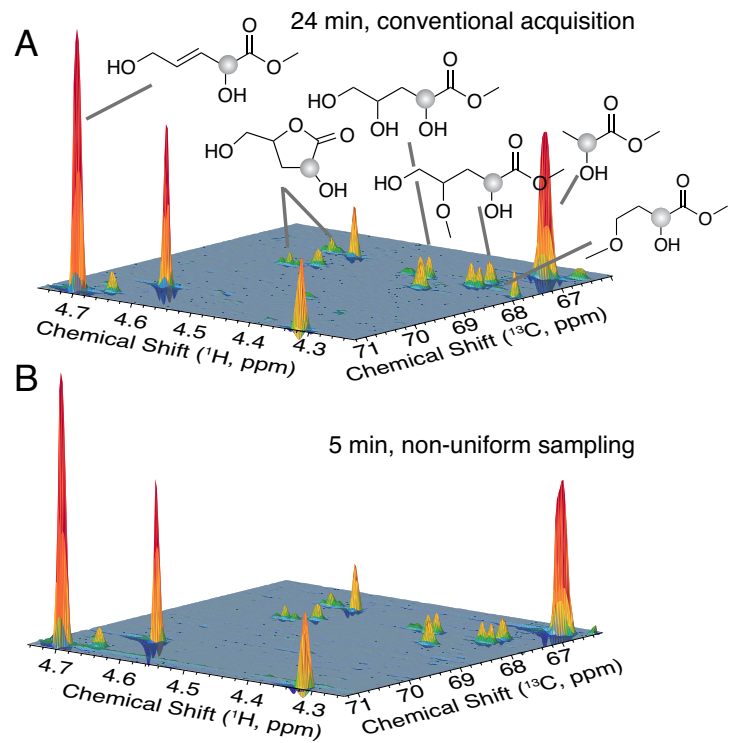

Figure 6. ${ }^{1} \mathrm{H}-{ }^{13} \mathrm{C}$ HSQC spectra obtained by a conventional (A) method employing 24 minutes acquisition time, and a non-uniform sampling method (B) using 5 minutes acquisition time.

\subsection{Validation}

To validate the demonstrated methods, a single reference sample of methyl lactate $(9.2 \mathrm{mg}$ of $98 \%$ nominal purity, $0.087 \mathrm{mmol}$ ) in methanol was prepared in the presence of $\mathrm{DMSO}_{2}$ as internal standard. A quantitative ${ }^{13} \mathrm{C}$ NMR spectrum and a ${ }^{1} \mathrm{H}$ ${ }^{13} \mathrm{C}$ HSQC NMR spectrum were recorded to determine the response factor of methyl lactate relative to $\mathrm{DMSO}_{2}$ in the HSQC spectrum. Subsequently, four samples were prepared of varying amounts of methyl lactate. Amounts of methyl lactate were determined by weighing with an analytical balance. Samples were prepared by dissolving the four samples in methanol solution in the presence of $\mathrm{DMSO}_{2}$. Quantitation with ${ }^{1} \mathrm{H}-{ }^{13} \mathrm{C}$ HSQC NMR was performed using the response factor calculated with a single independent calibration sample and was compared to gravimetric determinations (Figure 7A) and to quantitation by qNMR (Figure 7B). Linear regression between HSQC and gravimetric determinations yielded a Pearson correlation coefficient of 0.9995 and a slope of 0.998 . Linear regression between $\mathrm{HSQC}$ and quantitative ${ }^{13} \mathrm{C}$ NMR determinations yielded a Pearson correlation coefficient of 0.9997 and a slope of 1.016. Overall, comparison of the HSQC determinations using response factors with gravimetric and quantitative NMR determination show excellent consistency.
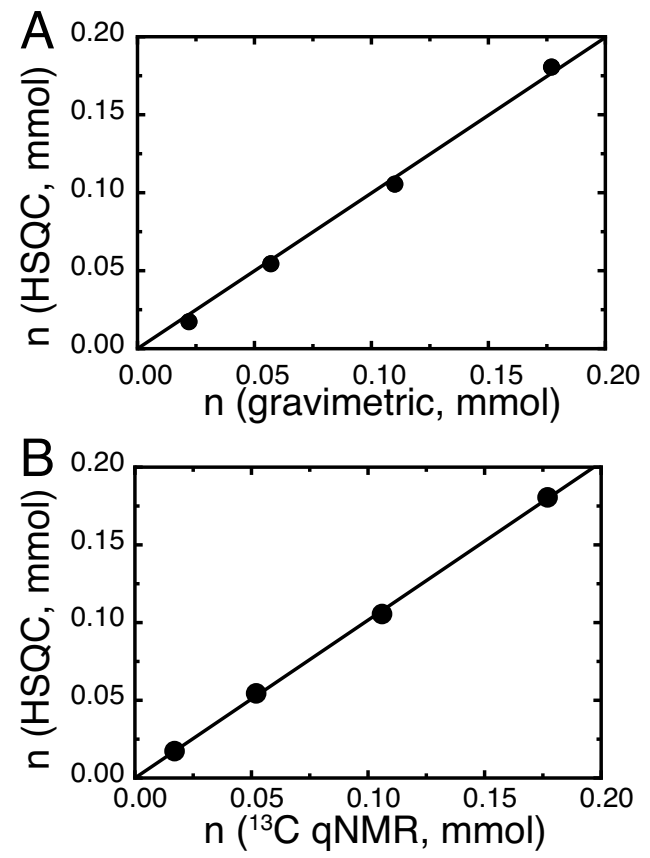

Figure 7. Comparison of methyl lactate amounts determined by ${ }^{1} \mathrm{H}-{ }^{13} \mathrm{C}$ HSQC with gravimetric analysis (A) and with quantitative ${ }^{13} \mathrm{C}$ NMR analysis (B). 


\section{Conclusion}

The cost and atom economy of sustainable processes with biomass depend on the efficient identification, quantification and exploitation of valuable chemicals in the post-reaction material. Where pure reference samples of new chemicals are not commercially available, 1D qNMR is a suitable method for the determination of concentrations, as the same number of nuclei in different chemical compounds produces equivalent signals. Hence, 1D qNMR spectra can be used to determine absolute concentrations in the absence of the purified reference compounds and can thereafter be used to obtain response factors in other analytical methods resolving the compounds of interest.

Here, we have shown that this approach is applicable for obtaining response factors relative to an internal standard for more sensitive and better resolved 2D NMR spectra. Following calibration, these 2D NMR spectra can be used to analyse samples within an experiment time of a few minutes. The higher sensitivity provided by 2D NMR spectra relative to 1D NMR spectra can be particularly beneficial for the identification of compounds at low concentrations, for instance in initial rate experiments, and for the quantification of low populated reaction intermediates.

The approach described herein relies on a correct quantification by 1D qNMR, while the subsequent 2D NMR spectrum merely needs to be acquired in a reproducible manner. Uncertainties in the acquisition of qNMR reference spectra have been described [31,35], where internally standardized spectra have less sources of uncertainty than externally standardized spectra [31]. The residual uncertainties that have to be taken into account include weighing uncertainties of the internal standards as well as its purity, including for instance water content, and potential degradation of the internal standards. Notably, these uncertainties in the amount of internal standard will elicit a relative error of determination.

In summary, the referencing of sensitive and rapid quantitative NMR spectra to quantitative onedimensional spectra permits screening biomass conversion reaction at attractive throughput rates and high chemical detail.

\section{Materials and Methods}

\subsection{Sample Preparation}

Test reactions were conducted as previously described [14]. NMR samples were prepared by rapid cooling of the reaction vessel to room temperature, removal of the Sn-Beta catalyst by filtration through a $0.4 \mu \mathrm{m}$ syringe filter and addition of $50 \mu \mathrm{d} 4$ methanol (Sigma Aldrich) as NMR lock substance to $500 \mu \mathrm{l}$ of the post-reaction material.

\subsection{NMR Spectroscopy}

All NMR spectra were acquired at $25^{\circ} \mathrm{C}$ on a Bruker Avance III $800 \mathrm{MHz}$ NMR instrument equipped with a TCI CryoProbe. Quantitative one-dimensional $\mathrm{C}$ NMR spectra were acquired by acquiring $64 \mathrm{k}$ complex data points of the $\mathrm{C}$ signal with a spectral width of $239 \mathrm{ppm}$, thus sampling an acquisition time of 1.36 seconds for the $\mathrm{C}$ free induction decay. 128 scans were acquired with an inter-scan recycle delay of 60 seconds. Inverse gated coupling was applied only during the acquisition period to avoid differential "C signal enhancements through polarization transfer from $\mathrm{H}$ to $\mathrm{C}$. " $\mathrm{C} \mathrm{T}$, relaxation times were probed by the inversion recovery method in order to validate that a recycle delay of 30 seconds suffices to allow return of the magnetization to its equilibration for quantitative determinations of protonated carbon atoms.

Two-dimensional $\mathrm{H} \mathrm{H}-\mathrm{C}$ HSQC spectra were recorded as data matrices of $1024 \times 300$ complex data points and a spectral width of 9 and $30 \mathrm{ppm}$ in the $\mathrm{H}$ and $\mathrm{C}$ dimensions, respectively. An inter-scan recycle delay of 1 second was employed. This experiment sampled the free induction decay for 142 $\mathrm{ms}$ in the $\mathrm{H}$ and for $50 \mathrm{~ms}$ in the $\mathrm{C}$ dimension, respectively. A relatively narrow spectral width in the "C dimension (55-85 ppm) was employed to rapidly achieve good spectral resolution in the $\mathrm{C}$ dimension as described previously [21,36,37]. Analyte signals and reference compound signals of interest are subject to spectral aliasing if their $\mathrm{C}$ chemical shifts occur outside the narrow "C NMR spectral width and appear at observed positions $\delta \mathrm{C}_{\mathrm{ab}}=\delta \mathrm{C}+/-\mathrm{n} * \mathrm{SW}$, where $\mathrm{n}$ is an integer and $\mathrm{SW}$ is the $\mathrm{C}$ spectral width of the 2D NMR spectrum. $\mathrm{H}-{ }^{-} \mathrm{C}$ HSQC NMR spectra were acquired within 24 minutes of experiment time. Non-uniform sampling of the indirect dimension [38] was subsequently used to accelerate data acquisition of the 2D NMR spectra. Spectra of acceptable quality were obtained with sampling of $20 \%$ of the data points in the indirect dimension, amounting to a time requirement for acquisition of $2 \mathrm{D} \mathrm{H}-\mathrm{C}$ HSQC 
spectra on post-reaction samples of 5 minutes per sample.

A 2D ${ }^{H-} \mathrm{C}$ HSQC spectrum without decoupling in the $\mathrm{C}$ dimension was acquired to probe the variation of $J_{с н}$ coupling constants at the $\mathrm{C} 2$ position of $\alpha$-hydroxy esters, showing a very narrow distribution of $J_{c н}$ values (146.0-146.8 Hz) with negligible influence on $2 \mathrm{D}$ NMR response factors. A 2D $\mathrm{H}-\mathrm{C}$ HSQC NMR spectrum with an inter-scan delay of 15 seconds was recorded to show that $\mathrm{H} \mathrm{T}_{\text {, }}$ relaxation has a predominant influence on differential response factors for the C-2 positions of different $\alpha$ hydroxy esters.

\subsection{Data Fitting}

$\mathrm{T}_{1}$ relaxation times were determined with the inversion-recovery method for methyl lactate and DPM, representing one of the smallest and one of the largest analytes in the current analysis. Signal areas $A(\tau)$ were recorded as a function of a relaxation delay $\tau$ and ${ }^{13} \mathrm{C} \mathrm{T}_{1}$ relaxation times at the $\mathrm{C}-2$ position of methyl lactate and DPM were determined as:

$$
\text { (4) } A(\tau)=A(0)\left[1-2\left(e^{-\frac{\tau}{T_{1}}}\right)\right]
$$

where $A(0)$ is the the signal area obtained from the Boltzmann magnetization at thermal equilibrium. Data were fit using the program proFit 6.2.9 (QuantumSoft, Zurich, Switzerland). $\mathrm{T}_{1}$ relaxation times indicate that recycle delays in the order of 30 seconds correspond to a delay longer than $5 \mathrm{~T}_{1}$ even for methyl lactate and hence permit the acquisition of quantitative ${ }^{13} \mathrm{C}$ NMR spectra (Supplementary Figure S2).

\section{Acknowledgements}

This work was funded by the Innovation Fund Denmark (case number 5150-00023B). $800 \mathrm{MHz}$ NMR spectra were recorded by using the spectrometer of the NMR center DTU supported by the Villum foundation.

\section{References}

1. Cherubini F (2010) The biorefinery concept: Using biomass instead of oil for producing energy and chemicals. Energy Conversion and Management 51 (7):1412-1421

2. Kokossis AC, Yang A (2010) On the use of systems technologies and a systematic approach for the synthesis and the design of future biorefineries. Computers \& Chemical Engineering 34 (9):13971405
3. Moreau C, Durand R, Razigade S, Duhamet J, Faugeras P, Rivalier P, Ros P, Avignon G (1996) Dehydration of fructose to 5-hydroxymethylfurfural over H-mordenites. Applied Catalysis A: General 145 (1):211-224

4. Dusselier M, Mascal M, Sels BF (2014) Top Chemical Opportunities from Carbohydrate Biomass: A Chemist's View of the Biorefinery. In: Nicholas KM (ed) Selective Catalysis for Renewable Feedstocks and Chemicals. Springer International Publishing, Cham, pp 1-40.

5. Antal MJ, Mok WSL, Richards GN (1990) Mechanism of formation of 5-(hydroxymethyl)-2furaldehyde from d-fructose and sucrose. Carbohydrate Research 199 (1):91-109

6. Holm MS, Saravanamurugan S, Taarning E (2010) Conversion of sugars to lactic acid derivatives using heterogeneous zeotype catalysts. Science 328 (5978):602-605

7. Moliner M, Roman-Leshkov Y, Davis ME (2010) Tin-containing zeolites are highly active catalysts for the isomerization of glucose in water. Proceedings of the National Academy of Sciences of the United States of America 107 (14):6164-6168

8. Zhao H, Holladay JE, Brown H, Zhang ZC (2007) Metal Chlorides in Ionic Liquid Solvents Convert Sugars to 5-Hydroxymethylfurfural. Science 316 (5831):1597

9. Choudhary V, Mushrif SH, Ho C, Anderko A, Nikolakis V, Marinkovic NS, Frenkel AI, Sandler SI, Vlachos DG (2013) Insights into the Interplay of Lewis and Brønsted Acid Catalysts in Glucose and Fructose Conversion to 5-(Hydroxymethyl)furfural and Levulinic Acid in Aqueous Media. Journal of the American Chemical Society 135 (10):3997-4006

10. Tolborg S, Meier S, Sádaba I, Elliot SG, Kristensen SK, Saravanamurugan S, Riisager A, Fristrup P, Skrydstrup T, Taarning E (2016) Tincontaining silicates: identification of a glycolytic pathway via 3-deoxyglucosone. Green Chemistry 18 (11):3360-3369

11. Markley JL, Brüschweiler R, Edison AS, Eghbalnia HR, Powers R, Raftery D, Wishart DS (2017) The future of NMR-based metabolomics. Current opinion in biotechnology 43:34-40

12. Hollis DP (1963) Quantitative Analysis of Aspirin, Phenacetin, and Caffeine Mixtures by 
Nuclear Magnetic Resonance Spectrometry. Analytical Chemistry 35 (11):1682-1684

13. Forseth RR, Schroeder FC (2011) NMRspectroscopic analysis of mixtures: from structure to function. Current opinion in chemical biology 15 (1):38-47

14. Elliot SG, Tolborg S, Sádaba I, Taarning E, Meier S (2017) Quantitative NMR Approach to Optimize the Formation of Chemical Building Blocks from Abundant Carbohydrates. ChemSusChem 10 (14):2990-2996

15. Kostidis S, Addie RD, Morreau H, Mayboroda OA, Giera M (2017) Quantitative NMR analysis of intra- and extracellular metabolism of mammalian cells: A tutorial. Analytica chimica acta 980:1-24

16. Lerche MH, Yigit D, Frahm AB, ArdenkjaerLarsen JH, Malinowski RM, Jensen PR (2018) Stable Isotope-Resolved Analysis with Quantitative Dissolution Dynamic Nuclear Polarization. Anal Chem 90 (1):674-678

17. Mutlib A, Espina R, Atherton J, Wang J, Talaat R, Scatina J, Chandrasekaran A (2012) Alternate strategies to obtain mass balance without the use of radiolabeled compounds: application of quantitative fluorine (19F) nuclear magnetic resonance (NMR) spectroscopy in metabolism studies. Chemical research in toxicology 25 (3):572-583

18. Simmler C, Napolitano JG, McAlpine JB, Chen SN, Pauli GF (2014) Universal quantitative NMR analysis of complex natural samples. Current opinion in biotechnology 25:51-59

19. Sette M, Wechselberger R, Crestini C (2011) Elucidation of lignin structure by quantitative 2D NMR. Chemistry 17 (34):9529-9535

20. Yan J, Kiemle D, Liu S (2015) Quantification of xylooligomers in hot water wood extract by $1 \mathrm{H}-13 \mathrm{C}$ heteronuclear single quantum coherence NMR. Carbohydrate polymers 117:903-909

21. Bøjstrup M, Petersen BO, Beeren SR, Hindsgaul O, Meier S (2013) Fast and accurate quantitation of glucans in complex mixtures by optimized heteronuclear NMR spectroscopy. Analytical Chemistry 85 (18):8802-8808

22. Li X, Hu K (2017) Chapter Three - Quantitative NMR Studies of Multiple Compound Mixtures. In: Webb GA (ed) Annual Reports on NMR Spectroscopy, vol 90. Academic Press, pp 85-143. doi:https://doi.org/10.1016/bs.arnmr.2016.08.001
23. Do NM, Olivier MA, Salisbury JJ, Wager CB (2011) Application of quantitative $19 \mathrm{~F}$ and $1 \mathrm{H}$ NMR for reaction monitoring and in situ yield determinations for an early stage pharmaceutical candidate. Anal Chem 83 (22):8766-8771

24. Rundlof T, Mathiasson M, Bekiroglu S, Hakkarainen B, Bowden T, Arvidsson T (2010) Survey and qualification of internal standards for quantification by $1 \mathrm{H}$ NMR spectroscopy. Journal of pharmaceutical and biomedical analysis 52 (5):645651

25. Rundlof T, McEwen I, Johansson M, Arvidsson T (2014) Use and qualification of primary and secondary standards employed in quantitative (1) $\mathrm{H}$ NMR spectroscopy of pharmaceuticals. Journal of pharmaceutical and biomedical analysis 93:111-117

26. Elliot SG, Taarning E, Madsen R, Meier S (2018) NMR Spectroscopic Isotope Tracking Reveals Cascade Steps in Carbohydrate Conversion by TinBeta. ChemCatChem 10 (6):1414-1419

27. Elliot SG, Tolborg S, Madsen R, Taarning E, Meier S (2018) Effects of Alkali-Metal Ions and Counter Ions in Sn-Beta-Catalyzed Carbohydrate Conversion. ChemSusChem 11 (7):1198-1203

28. Saravanamurugan S, Tosi I, Rasmussen K, Jensen R, Taarning E, Meier S, Riisager A (2017) Facile and Benign Conversion of Sucrose to Fructose Using Zeolites With Balanced Brønsted and Lewis Acidity. Catalysis Science \&Technology 7: 2782-2788

29. Tosi I, Riisager A, Taarning E, Jensen P, Meier S (2018) Kinetic Analysis of Hexose Conversion to Methyl Lactate by Sn Beta: Effects of Substrate Masking and of Water. Catalysis Science \& Technology 8: 2137-2145

30. Cullen CH, Ray GJ, Szabo CM (2013) A comparison of quantitative nuclear magnetic resonance methods: internal, external, and electronic referencing. Magnetic resonance in chemistry : MRC 51 (11):705-713

31. Le Gresley A, Fardus F, Warren J (2015) Bias and Uncertainty in Non-Ideal qNMR Analysis. Critical Reviews in Analytical Chemistry 45 (4):300310

32. Elliot SG, Andersen C, Tolborg S, Meier S, Sádaba I, Daugaard AE, Taarning E (2017) Synthesis 
of a novel polyester building block from pentoses by tin-containing silicates. RSC Advances 7 (2):985-996

33. Webster GK, Marsden I, Pommerening CA, Tyrakowski CM, Tobias B (2009) Determination of relative response factors for chromatographic investigations using NMR spectrometry. Journal of pharmaceutical and biomedical analysis 49 (5):12611265

34. Petersen BO, Hindsgaul O, Meier S (2014) Profiling of carbohydrate mixtures at unprecedented resolution using high-precision $1 \mathrm{H}-13 \mathrm{C}$ chemical shift measurements and a reference library. Analyst 139 (2):401-406

35. Malz F, Jancke H (2005) Validation of quantitative NMR. Journal of pharmaceutical and biomedical analysis 38 (5):813-823

36. Vitorge B, Jeannerat D (2006) NMR diffusion measurements in complex mixtures using constanttime-HSQC-IDOSY and computer-optimized spectral aliasing for high resolution in the carbon dimension. Anal Chem 78 (15):5601-5606

37. Njock GB, Pegnyem DE, Bartholomeusz TA, Christen P, Vitorge B, Nuzillard JM, Shivapurkar R, Foroozandeh M, Jeannerat D (2010) Spectral aliasing: a super zoom for 2D-NMR spectra. Principles and applications. Chimia 64 (4):235-240

38. Kazimierczuk K, Orekhov VY (2011) Accelerated NMR Spectroscopy by Using Compressed Sensing. Angewandte Chemie International Edition 50 (24):5556-5559 